\title{
Piezo-Type Mechanosensitive Ion Channel Component 2
}

National Cancer Institute

\section{Source}

National Cancer Institute. Piezo-Type Mechanosensitive Ion Channel Component 2. NCI Thesaurus. Code C106088.

Piezo-type mechanosensitive ion channel component 2 (2752 aa, $\sim 318 \mathrm{kDa}$ ) is encoded by the human PIEZO2 gene. This protein is involved in ion transport. 